\title{
PLAN DE NEGOCIOS DE UN CIRCUITO AGROTURÍSTICO DEL CAFÉ PLUMA COMO ESTRATEGIA DE DESARROLLO TERRITORIAL EN EL MUNICIPIO DE PLUMA HIDALGO, OAXACA, MÉXICO
}

\section{BUSINESS PLAN OF AN AGRITURISTIC CIRCUIT OF THE COFFEE BOARD AS A STRATEGY OF TERRITORIAL DEVELOPMENT IN THE MUNICIPALITY OF PLUMA HIDALGO, OAXACA, MEXICO}

\section{Colin-Rodea, D. ${ }^{1}$; Thomé Ortiz, H. ${ }^{1}$; Ávalos de la Cruz, D.A. ${ }^{2}$}

\begin{abstract}
${ }^{1}$ Universidad Autónoma del Estado de México, Instituto de Ciencias Agropecuarias y Rurales. el Cerrillo, Piedras Blancas, Toluca, Estado de México. ${ }^{2}$ Colegio de Postgraduados, Campus Córdoba. Carretera Federal Córdoba-Veracruz km 348, Manuel León, Amatlán de los Reyes, Veracruz, México.
\end{abstract}

*Autor de correspondencia: humbertothome@hotmail.com

\begin{abstract}
During the period from March 2015 to November 2016, an empirical case study was conducted, with a participatory approach, in the municipality of Pluma Hidalgo, Oaxaca, Mexico. The aim was to propose a business plan for productive diversification of coffee (Coffea arabica L.) production through agro-tourism. The results show that there is a base of natural and cultural resources, a collective organization, an emblematic resource linked to the territory (coffee Pluma), a network of goods and services and a potential market that determine the feasibility of the agrotourism proposal. It is concluded that planning is a substantive activity for the development of the agrotourism business and that this must be shared by various sectors of society that are interested, among which is the community, academia, public institutions and conscious tourists.
\end{abstract}

Keywords: Rural development, rural tourism, coffee, Oaxaca.

\section{RESUMEN}

Durante el periodo de marzo del año 2015 a noviembre de 2016 se desarrolló un estudio de caso, de carácter empírico con enfoque participativo en el municipio de Pluma Hidalgo, Oaxaca, México. El objetivo fue proponer un plan de negocios para la diversificación productiva de la producción de café (Coffea arabica L.), a través del agroturismo. Los resultados muestran que existe una línea base de recursos naturales y culturales, una organización colectiva, un recurso emblemático ligado al territorio (café Pluma), un entramado de bienes y servicios y un mercado potencial que determinan la factibilidad de la propuesta agroturística. Se concluye que la planificación es una actividad sustantiva para el desarrollo del negocio agroturístico y que esta debe ser compartida por diversos sectores de la sociedad que resulten interesados, entre los que se encuentra la comunidad, la academia, las instituciones públicas y los turistas responsables.

Palabras clave: Desarrollo rural, turismo rural, café, Oaxaca. 


\section{INTRODUCCION}

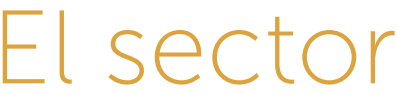

productor de café (Coffea arabica L.) en México, atraviesa por momentos críticos debido a varios factores El calentamiento global, las plagas, los bajos precios de grano en el mercado internacional y la falta de política sectorial que permitan mantener sus tasas de crecimiento, han contribuido a la disminución de la actividad cafetalera y al encarecimiento de la calidad de vida de las comunidades cafetaleras.

Durante los últimos años la falta de producción se ha sustituido por café importado de menor precio y calidad, principalmente de variedad robusta. Lo anterior, golpea directamente a los productores mexicanos, en especial a los de variedades de mejor calidad quienes ante esta situación recurren a la sustitución del café por otros cultivos más rentables, la migración y el abandono del campo.

La producción de café en México se lleva a cabo, en su mayoría, por poblaciones indígenas. En la región de Pluma Hidalgo, Oaxaca se cultiva café orgánico de alta calidad y características únicas. Actualmente, los cafeticultores indígenas viven en condiciones precarias debido a las deficientes políticas públicas que los dejan al margen de la actividad comercial, la disminución en el cultivo de café en esta zona repercute a nivel económico, cultural, social, en la biodiversidad, calidad de los suelos y paisajes.

La difícil situación orilló a los cafeticultores de Pluma Hidalgo a emprender acciones para contrarrestar los efectos de la crisis cafetalera, las iniciativas de carácter turístico aparentemente resultaron alternativas viables que, aunque pobremente planeadas, han contribuido a diversificar las actividades económicas, aspecto que hace evidente la necesidad de estructurar un plan de negocios que permita una mejor gestión de las actividades turísticas.

La preservación de las actividades tradicionales de un pueblo contribuye a la conservación de su identidad, para lo que es necesaria la revalorización de los elementos identitarios de las comunidades que corren peligro en el contexto de la globalización económica y cultural. Las condiciones de vida de estas poblaciones han sido determinadas por el territorio que habitan, pero la falta políticas públicas y las directrices que marca el gobierno para el desarrollo de las comunidades rurales, han difi- cultado la dinamización del sector agrícola, la situación se agrava si consideramos que la creciente pobreza en las zonas rurales como Pluma Hidalgo ocasionan la pérdida de los elementos que componen el patrimonio natural y cultural. Acciones alternativas como el desarrollo del agroturismo planificado podrían convertir al cultivo del café en un factor dinamizador del desarrollo en la comunidad.

El planteamiento de proyectos de desarrollo local, desde una perspectiva turística, tal como el agroturismo o el turismo agroalimentario pueden llegar a permitir que un producto agrícola, característico de una región, se convierta en un catalizador de procesos de desarrollo local en la población, se revaloriza el territorio, se diversifican las actividades económicas y se da un desarrollo integral del lugar a partir de los recursos propios de la región.

En el municipio de Pluma Hidalgo, el desarrollo de proyectos planificados de turismo rural permitiría no solo el rescate de su patrimonio, sino que también podría mitigar la migración de los habitantes de este municipio a otros lugares, con el fin de mejorar sus condiciones de vida. Con el desarrollo de un circuito agroturístico del café Pluma, se pueden generar empleos y la inclusión de los sectores marginados del sistema capitalista, lo que convierte al turismo rural en diversificador de ingresos y eje del desarrollo de la comunidad rural.

El objetivo del trabajo fue desarrollar un plan de negocios para la diversificación productiva de café en la región, a través de una perspectiva horizontal y un enfoque de investigación acción participativa. Para ello el documento se integró de la siguiente manera: después de este apartado introductorio se presenta un breve resumen del trabajo, posteriormente se explican los materiales y métodos usados en la investigación, más adelante se presentan los resultados obtenidos y la propuesta del plan de negocios, finalmente se exponen las condiciones finales.

En este contexto, el objetivo de esta investigación fue proponer un plan de negocios con enfoque participativo, para la diversificación productiva del café Pluma, de Pluma Hidalgo, Oaxaca, a través del agroturismo como una actividad económica complementaria.

\section{MATERIALES Y MÉTODOS}

La presente investigación se desarrolló a partir de la metodología de estudio de caso simple, con un enfoque 
mixto, es decir que se recuperaron datos cualitativos y cuantitativos sobre la unidad de observación seleccionada. De acuerdo con Stake (1998), esta metodología permite captar la particularidad y la complejidad de un caso singular, para comprender su actividad en circunstancias concretas. Su relevancia radica en la interpretación del caso a partir de sus características particulares, a través de un examen holista de la singularidad, lo que significa tener en cuenta las complejidades que lo determinan y definen (Stake, 1998).

El procedimiento desarrollado se ajustó a las etapas definidas por (Yin, 1994) que son: i) delimitación del problema; ii) diseño de la investigación; iii) recolección y análisis de datos; y iv) interpretación de los resultados. Se trató de una investigación empírica que abordó un fenómeno contemporáneo dentro de su contexto original.

La selección y delimitación del caso se dio en función de los siguientes criterios:

i) Consideración del contexto socioeconómico marginal de Pluma Hidalgo, Oaxaca, que hiciera evidente la necesidad de plantear estrategias de desarrollo local

ii) La existencia de una producción agroalimentaria diferenciada, anclada al territorio.

iii) Que fuera un territorio de características, naturales y culturales únicas con potencial para su aprovechamiento turístico.

Los criterios anteriores se consideraron debido a que el presente trabajo de investigación busca identificar las posibles intersecciones entre la producción de café y el turismo agroalimentario como estrategia de desarrollo territorial.

\section{Herramientas}

Las herramientas empleadas en esta investigación se explican en la Figura 1.

Se realizó la caracterización del territorio de Pluma Hidalgo para evaluar la condición de los recursos naturales, la población y las actividades productivas. De acuerdo con el Instituto Nacional de Ecología (INE, 2000), un diagnóstico responde a la pregunta: ¿cómo está?, para determinar la factibilidad de una propuesta de desarrollo que contemple el equilibrio entre el estado natural, la presión social y productiva del territorio.

A partir de lo anterior se realizó un análisis FODA con enfoque participativo (Rosas, 2001), para establecer, colectivamente, cuál sería la posición estratégica para abordar el proyecto y cuáles serían las acciones a llevar a cabo en las distintas áreas involucradas en la propuesta del plan de negocios del circuito agroturístico.

La muestra consistió en 20 productores de café que fueron determinados mediante el método muestreo no probabilístico de bola de nieve (Pérez, 2000). Igualmente, fueron atendidos los principios del muestreo por conveniencia (Rubio, 2005), considerando que la incorporación al proyecto fue exclusivamente de aquellos actores que mostraron voluntad en participar.

\section{RESULTADOS Y DISCUSIÓN}

\section{Diagnostico del territorio}

El diagnóstico del territorio se realizó con base en la información recabada a través de la aplicación de cédulas en las cuales se recolectó información geográfica, poblacional, económica, cultural y social del municipio de Pluma Hidalgo (Cuadro 1).

Con la información recabada se demostró que Pluma Hidalgo posee una oferta considerable de recursos naturales y socioculturales con vocación turística, con características únicas en el segmento de las zonas cafetaleras, lo que le otorga un gran potencial para el desarrollo de proyectos turísticos en la región.

\section{Análisis FODA}

A continuación, se presentan los resultados del análisis FODA realizado como estrategia de diagnóstico con
Figura 1. Herramientas de investigación para llevar a cabo el plan de negocios de un circuito agroturístico del café Pluma como estrategia de desarrollo territorial en el municipio de Pluma Hidalgo, Oaxaca, México. 


\begin{tabular}{|c|c|c|c|c|}
\hline Medio Ambiente & $\begin{array}{c}\text { Población, Actividad } \\
\text { Económica, Comercios y } \\
\text { Servicios }\end{array}$ & $\begin{array}{c}\text { Cultura, Animación } \\
\text { Cultural }\end{array}$ & Ocio/ Deportes & Alojamiento /Restauración \\
\hline $\begin{array}{l}\text { *Cuerpos de agua } \\
\text { (cascada arcoíris, } \\
\text { cuencas del rio Copalita } \\
\text { y Tonameca, cascadas } \\
\text { Llano Grande } \\
\text { *Áreas naturales } \\
\text { protegidas (flora y fauna } \\
\text { endémica)> Finca el } \\
\text { Pacífico. }\end{array}$ & $\begin{array}{l}\text { *Aspectos Demográficos } \\
\text { (INEGI, 2007) } \\
\text { *Comercios Activos } \\
\text { *Infraestructura Existente } \\
\text { y sus condiciones } \\
\text { (carreteras, accesos, } \\
\text { transito). }\end{array}$ & $\begin{array}{l}\text { *Patrimonio histórico y } \\
\text { cultural de Pluma Hidalgo } \\
\text { *Lugares de interés para } \\
\text { los turistas } \\
\text { *Actividades culturales } \\
\text { relevantes del lugar } \\
\text { *Café Pluma como } \\
\text { espacialidad gastronómica }\end{array}$ & $\begin{array}{l}\text { *Zonas de baño } \\
\text { *Deportes ecuestres } \\
\text { ^Excursiones pedestres/ } \\
\text { ciclismo }\end{array}$ & $\begin{array}{l}\text { *Establecimientos } \\
\text { de alojamiento y sus } \\
\text { características. } \\
\text { *Establecimientos de } \\
\text { Alimentos y Bebidas y sus } \\
\text { características }\end{array}$ \\
\hline
\end{tabular}

el objetivo de determinar los factores principales involucrados en la planeación del proyecto del circuito agroturístico del café Pluma como un negocio. En este análisis se puntualizan las fortalezas y oportunidades que la idea de negocio tiene y las debilidades y amenazas que el proyecto tendría que contemplar dentro del desarrollo de su plan estratégico. Lo anterior con el objetivo de tener un negocio viable y adecuado a los recursos y el contexto actual del mismo (Figura 2).

Con los datos obtenidos después de haber realizado el análisis FODA se concluye que sus mayores fortalezas radican en la actividad cafetalera consolidada en la zona, en conjunto con la combinación de atributos naturales y culturales que ésta posee. Las amenazas para el proyecto provienen de la situación política y social del país, cuyo mayor problema es la inseguridad que afecta, también, a las zonas turísticas y que incide directamente en el flujo de turistas con destino hacia la sierra sur y a la zona de la costa de Oaxaca. Lo anterior, es de suma importancia debido a que parte de la estrategia de captación de flujos turísticos para el circuito agroturístico del café Pluma contempla a los turistas de Huatulco y de Puerto Escondido. Por lo anterior una disminución en la afluencia de los turistas a la costa del estado de Oaxaca repercutiría directamente en una menor cantidad de turistas potenciales para el proyecto.

Es necesaria la planeación estratégica en manos de personas capacitadas en proyectos similares, pues con
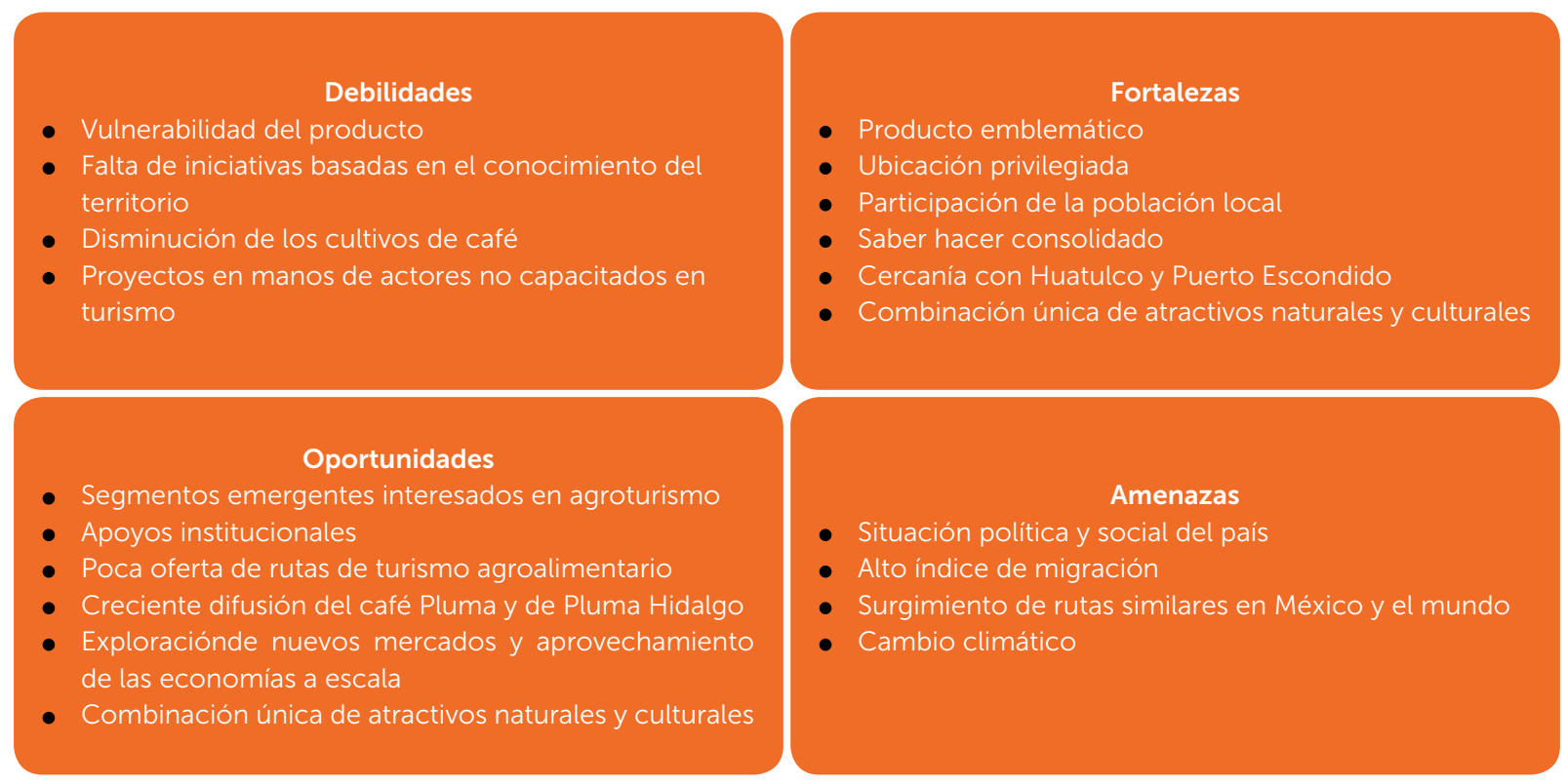

Figura 2. Análisis de Fortalezas, Oportunidades, Debilidades y Amenazas (FODA) como estrategia de diagnóstico con el objetivo de determinar los factores principales involucrados en la planeación del proyecto del circuito agroturístico del café Pluma como un negocio en el municipio de Pluma Hidalgo, Oaxaca, México.

- Segmentos emergentes interesados en agroturismo

- Apoyos institucionales

- Poca oferta de rutas de turismo agroalimentario

- Creciente difusión del café Pluma y de Pluma Hidalgo

- Exploraciónde nuevos mercados y aprovechamiento de las economías a escala

- Combinación única de atractivos naturales y culturales

- Producto emblemático

- Ubicación privilegiada

- Participación de la población local

- Saber hacer consolidado

- Cercanía con Huatulco y Puerto Escondido

- Combinación única de atractivos naturales y culturales
- Situación política y social del país

- Alto índice de migración

Surgimiento de rutas similares en Mexico y el mundo

- Cambio climático 
este análisis se pudo observar que dentro de las debilidades del proyecto la falta de capacitación y el desconocimiento del territorio son variables presentes y que habría que atender de manera prioritaria con el objetivo de gestionar un proyecto benéfico para la población y que contribuya al desarrollo de la localidad a largo plazo.

\section{Plan de negocios}

El plan de negocios se desarrolló a partir de la información recabada en la revisión documental y con los datos arrojados por el trabajo de campo. Se planteó, con base en el contexto y características del lugar, tomando en consideración el aspecto prioritario del desarrollo local sustentable. Para la elaboración de la propuesta del plan de negocios del Circuito Agroturístico del Café Pluma se realizó, en primer lugar, un análisis de la oferta, la demanda y de las tendencias del mercado. A continuación, se llevó a cabo el planteamiento de la idea de negocio a través del desarrollo de un modelo CANVAS (Alario-Hoyos et al., 2014) como estrategia para la identificación de los actores, elementos involucrados y funciones a realizar. Posteriormente se realizó un estudio de mercado en el cual se detecta una demanda emergente de turistas provenientes de la zona de Huatulco y Puerto Escondido en búsqueda de actividades alternativas al turismo de sol y playa. Se identificaron dos segmentos de turistas, nacional y extranjero, aunque el primero es sensible al precio, ambos interesados en conocer la cultura, la historia e interactuar con la forma de vida de la localidad para vivir nuevas experiencias.

Para el diseño de un circuito agroturístico, a partir del cultivo del café Pluma del municipio de Pluma Hidalgo, Oaxaca, se llevó a cabo el análisis de las rutas de los productos de turismo agroalimentario que representan una competencia directa debido a que están basados en producciones diferenciadas de café en el país, se identificaron: la Ruta de las Fincas Cafetaleras del Soconusco en Chiapas, la Ruta del Café y la Vainilla y la Ruta Aventura del Café, ambas en el estado de Veracruz. Al realizar un análisis comparativo podemos apreciar las similitudes presentes entre los proyectos de rutas cafetaleras: infraestructura, recursos, logística, capital social y político. Con base en lo anterior también podemos identificar las similitudes y diferencias con el proyecto de circuito agroalimentario del café Pluma: por una parte, la ubicación y las características del territorio dan como resultado un lugar de riqueza natural, contenedor de gran biodiversidad y riqueza paisajística, cuenta con montañas, cascadas y diversas plantas endémicas.
La variedad de recursos con los que cuenta esta comunidad le brindan la oportunidad de generar una oferta diversa de actividades de turismo rural, lo cual resulta positivo pues se encuentra rodeado de destinos turísticos ya consolidados y con una infraestructura ya desarrollada como Puerto Escondido y Huatulco, que reciben turismo de sol y playa y San José del Pacífico que recibe turismo de aventura y de naturaleza, además de poblaciones semi urbanizadas, capaces de proveer de transporte, alimentos y todo tipo de insumos y requerimientos necesarios para el desarrollo de la actividad turística.

Sumado a lo anterior otra ventaja es el interés de la población por participar en la actividad turística como un medio para mejorar su calidad de vida, a través de la obtención de mejores ingresos y para lograr el reconocimiento y la continuidad de la actividad cafetalera, a través de la difusión de la tradición que distingue al lugar. Este interés ha provocado iniciativas individuales por parte de finqueros quienes, con capital propio o con el apoyo de inversionistas privados han hecho de sus fincas, proyectos agroturísticos improvisados, algunos más sofisticados que otros, que han logrado captar turistas paulatinamente y colocar a Pluma Hidalgo como un destino de turismo rural. Esta autogeneración de proyectos, a nivel individual, ha conducido también a la creación de una oferta diversa capaz de captar turistas con diferentes presupuestos e intereses, lo cual representa una ventaja a la hora de hacer un recuento de las características particulares del proyecto del circuito agroturístico del café Pluma, ante las otras rutas que configuran la competencia.

Se realizó, también, un estudio técnico en el cual se establecieron, de manera detallada, los procesos y requerimientos del proyecto del circuito. Este estudio sirvió, junto con el estudio administrativo, como base para el posterior estudio financiero que tuvo a bien mostrar la estructura de costos y la rentabilidad del negocio, en materia financiera. Describiendo la inversión inicial, la fuente de financiamiento, estados financieros proforma y el punto de equilibrio, para proporcionar una información más certera sobre el aspecto financiero de la empresa y su crecimiento.

La inversión inicial se compone de los gastos destinados a mobiliario y equipo, gastos de constitución y el capital de trabajo equivalente a 3 meses de gastos fijos y sueldos. 
La inversión necesaria para este proyecto cubre los gastos preoperativos y los operativos de los tres primeros meses, lo anterior con un monto de $\$ 275,322.13$ pesos mexicanos. El dinero para el financiamiento del proyecto se pretende obtener de dos fuentes: la primera parte proviene de la cuota de inscripción que paguen los productores y microempresarios por afiliarse al circuito agroturístico del café Pluma, en un principio se contemplan 20 socios y este dinero se emplearía para cubrir los gastos del inicio del proyecto y posteriormente estará destinado al mantenimiento de la ruta. La segunda parte del financiamiento que se ocupará para el arranque del circuito, se pretende gestionar a través del gobierno municipal, ante la Secretaria de Turismo, como apoyo a la iniciativa turística en el municipio. Actualmente, las pequeñas empresas ya reciben apoyos del municipio, por lo cual, al formalizar y constituir la figura de la sociedad cooperativa integrada por los diversos gremios del municipio, el financiamiento municipal representa una opción viable para el proyecto.

\section{CONCLUSIONES}

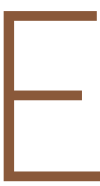

I turismo en las zonas cafetaleras ha traído consigo, por un lado, el surgimiento de actividades económicas alternativas a la producción de café las cuales han contribuido a la valorización del patrimonio natural y cultural, pero por otro lado la falta de planeación y conocimiento del territorio han generado daños al ecosistema. Debido a lo anterior, es importante que el turismo en zonas como Pluma Hidalgo, responda a modelos planificados de turismo con enfoque territorial, a partir del impulso de actividades rurales no agrícolas, complementarias a la producción de café.

Los resultados muestran que el turismo agroalimentario representa una alternativa viable para el desarrollo económico en el municipio de Pluma Hidalgo. La existencia de la variedad de café criolla Pluma Hidalgo, catalogada entre las tres mejores del mundo, representa una ventaja comparativa para la propuesta.

El diagnóstico realizado arrojó una serie de datos que, en definitiva, dan cuenta del potencial turístico del municipio de Pluma Hidalgo, además se pudieron identificar elementos de los cuales el proyecto pudiera echar mano con el objetivo de generar un proyecto sustentable y competitivo que resulte atractivo al mercado.

Ciertamente, la existencia de un producto diferenciado, fuertemente anclado al territorio, puede constituir el eje de un proyecto agroturístico en la región, pero para alcanzar los objetivos de valorización turística es necesaria una visión de planificación integral, donde se incorporen aspectos de asociatividad, modelos comerciales, conocimiento de la demanda y estrategias de mercado (Szmulewicz et al., 2012). Aspectos que frecuentemente escapan de las competencias desarrolladas por los productores agrícolas, quienes deben vincularse con otros actores especializados a partir del trabajo realizado por las instituciones, universidades y centros de investigación.

\section{LITERATURA CITADA}

Alario-Hoyos C., Pérez-Sanagustín M., Cormier D., Delgado-Kloos C. 2014. Proposal for a conceptual framework for educators to describe and design MOOCs. Journal of Universal Computer Science 20: 6-23

Instituto Nacional de Ecología (INE). 2000. Ordenamiento Ecológico General del Territorio. Memoria Técnica 1995-2000. SEMARNAP-INE. México, D. F

Instituto Nacional de Estadística y Geografía (INEGI). 2007. Prontuario de información geográfica municipal de los Estados Unidos Mexicanos. 18 de enero 2016, de INEGI Sitio web: http://www3.inegi.org. $\mathrm{mx} /$ sistemas/mexicocifras/datosgeograficos/20/20071.pdf

Stake R.E. 1998. Investigar con estudios de caso. Madrid, España: Ediciones Morata

Szmulewicz E.P., Gutiérrez V.C., Winkler C.K. 2012. Asociatividad y agroturismo. Evaluación de las habilidades asociativas en redes de agroturismo del sur de Chile. Estudios y Perspectivas en Turismo 21: 1013-1034

Pérez C. 2000. Técnicas de muestreo estadístico: Teoría, práctica y aplicaciones. México, D. F.: Alfaomega.

Rubio A. 2005. Técnicas de Muestreo. Lima, Perú: Universidad Nacional Agraria La Molina

Rosas J.C. 2001. Aplicación de metodologías participativas para el mejoramiento genético de frijol en Honduras. Agronomía Mesoamericana 12: 219-228.

Yin R. 1994. Case Study Research. Design and Methods. Applied Social Research Methods Series Vol. 5. Thousand Oaks. London, UK: Sage Publications. 